\title{
Reactividad Química de los Azo Colorantes Amarillo Anaranjado y Rojo Allura mediante Descriptores Globales y la Función de Fukui
}

Adolfo E. Ensuncho, Jesús M. López y Juana Robles

Universidad de Córdoba, Departamento de Química, Grupo de Química Computacional, Cra 6 № 74-103, Córdoba-Colombia (e-mail: adem9abril@hotmail.com; jmanuellopez.gqc@gmail.com, juanarobles2003@yahoo.com)

Recibido Abr. 18, 2012; Aceptado May. 29, 2012; Versión final recibida Jun. 07, 2012

\begin{abstract}
Resumen
Se calcularon descriptores globales y locales de la reactividad para los colorantes amarillo anaranjado y rojo allura con el fin de analizar su reactividad química intrínseca. Los cálculos computacionales se llevaron a cabo tanto en vacio como en la fase acuosa, la cual se representó por medio de una constante dieléctrica de 78.5 e introducida por el modelo de solvente del continuo polarizado. Los resultados mostraron que el colorante rojo allura es menos susceptible a la adición nucleofílica que el amarillo anaranjado, ya que los sustituyentes electrodonadores metilo y metoxilo disminuyen la capacidad del grupo azo para aceptar electrones. El análisis de la función condensada de Fukui para el ataque nucleofílico e índice de electrofilicidad local, mostraron que el sitio preferido de ataque del agente reductor (ión bisulfito) fue $\mathrm{C} 4$, lo cual estuvo en concordancia con los reportes experimentales.
\end{abstract}

Palabras clave: función de Fukui, azo colorantes, descriptores de la reactividad, cálculos DFT, modelo PCM

\section{Chemical Reactivity of the Azo dyes Sunset Yellow and Allura Red by Global Descriptors and the Fukui Function}

\begin{abstract}
In the present work local and global descriptors of the reactivity were calculated for sunset yellow and allura red to analyze its intrinsic reactivity. The computational calculations were carried out in vacuum and aqueous phase. The aqueous phase is represented by a dielectric constant of 78.5 , introduced by polarizable continuum model. The results show that allura red is less susceptible to nucleophilic addition than sunset yellow, because the electrodonor substituents methyl and methoxy decrease the capability of the azo group to accept electrons. The analysis of condensed Fukui function for a nucleophilic attack and local electrophilicity index showed that the preferred direction of attack the reducing agent (bisulfite ion) occurs on $\mathrm{C} 4$, which was in good agreement with experimental reports.
\end{abstract}

Keywords: function Fukui, azo dyes, reactivity descriptors, DFT calculations, PCM model 


\section{INTRODUCCIÓN}

Los colorantes azoicos de origen sintético, rojo allura (E-129, C.I. 16035) y amarillo anaranjado (E110, C.I.15985) son ampliamente usados en la industria de alimentos (Scotter y Castle, 2004; Marmion,1979) y se pueden encontrar en postres, gelatinas, jugos de frutas comerciales, aperitivos, entre otros (Ustun, 2004). A la mayoría de estos productos, se les agrega el conservante bisulfito de sodio con el fin de garantizar su durabilidad (Winkler et al., 2006), pero se ha observado que en algunos jugos de frutas comerciales conforme transcurre el tiempo la tonalidad inicial va desapareciendo ocasionando con ello que el consumidor pierda interés en adquirir el producto, ya que el color crea expectativas del sabor y la calidad en los alimentos (Umer et al., 2008). La evidencia experimental sugiere que la decoloración se debe al ión bisulfito que participa en este proceso mediante una reacción de adición nucleofílica (Wedzicha y Rumbelow, 1981). De ahí que, a finales de los ochenta algunos estudios al respecto fueron desarrollados, monitoreando la velocidad de degradación del colorante amarillo anaranjado por técnicas de espectrometría de masas por bombardeo con átomos rápidos y cromatografía líquida de alta resolución, encontrándose que efectivamente en presencia del ión bisulfito se formaba un compuesto coloreado amarillo-limón (Damant et al.,1989). Asimismo, por subsecuentes estudios de resonancia magnética nuclear y espectrometría de masas se mostró que la adición nucleofílica del ión bisulfito ocurría sobre el carbono en la posición 4 de la molécula de amarillo anaranjado (Damant et al., 1989). Por lo anterior es claro que, un conocimiento detallado de la de la reactividad química intrínseca de estos colorantes es clave, puesto que no solo permite la comprensión del fenómeno desde el punto de vista químico, sino además para buscar alternativas de solución frente a los procesos de degradación del color de estas especies. En este sentido, la química computacional se muestra atractiva, pues mediante la aplicación de sus formalismos se puede obtener información relevante sobre las propiedades y estructura electrónica de las moléculas de interés (Moncada y Salgado, 2007; Mendoza y Ríos, 2011; Adamson et al., 2008; Esquivel et al., 2010). Además es de nuestro conocimiento que los estudios mecanocuánticos sobre azo colorantes usando descriptores de la reactividad globales y locales son escasos (Özen et al., 2003; 2004). Por lo tanto, el presente trabajo busca contribuir a ganar una mayor comprensión del comportamiento químico de estos importantes colorantes usados en la industria de alimentos.

\section{METODOLOGÍA}

\section{Fundamentos Teóricos}

En el marco conceptual de la Teoría de Funcionales de la Densidad (DFT, por sus siglas en inglés), es posible obtener descriptores de la reactividad tales como; potencial químico electrónico $(\mu)$, dureza global $(\eta)$ e índice de electrofilicidad global $(\omega)$. En esta teoría, la energía puede expresarse en términos del número de electrones $\mathrm{N}$ y el potencial externo $\mathrm{v}(\mathrm{r})$, de modo que, $E[\rho(r)]$, también se puede expresar como, $E[N, v(r)]$. Las derivadas de $E[N, v(r)]$ con respecto al número de electrones y potencial externo producen el conjunto de descriptores globales ya mencionados (Mendoza y Ríos, 2011). El potencial químico electrónico, es una propiedad global que caracteriza la tendencia de los electrones a escapar de un sistema en equilibrio, mientras que la dureza global puede ser concebida como la resistencia a la transferencia de carga del sistema (Vivas-Reyes et al., 2008). Las cantidades $\mu$ y $\eta$, pueden ser calculadas con base en la aproximación de diferencias finitas de tres puntos y el teorema de Koopmas (Nelsen et al., 2007; Plakhutin y Davidson, 2009), como se indica en las ecuaciones 1 y 2 respectivamente.

$$
\begin{aligned}
& \mu=\frac{1}{2}\left(\varepsilon_{\mathrm{L}}+\varepsilon_{\mathrm{H}}\right) \\
& \eta=\frac{1}{2}\left(\varepsilon_{\mathrm{L}}-\varepsilon_{\mathrm{H}}\right)
\end{aligned}
$$

Donde $\varepsilon_{\mathrm{H}}$ y $\varepsilon_{\mathrm{L}}$ son las energías de los orbitales moleculares frontera más alto ocupado y más bajo desocupado (HOMO y LUMO, por sus siglas en inglés) respectivamente. El índice de electrofilicidad global puede determinarse a partir de la relación entre el potencial químico y la dureza como se muestra en la ecuación 3: 


$$
\omega=\frac{\mu^{2}}{2 \eta}
$$

El índice $\omega$, representa la energía de estabilización del sistema cuando este es saturado por electrones que provienen de los alrededores, es decir mide la tendencia de las especies químicas a recibir electrones (Chattaraj y Giri, 2009; De Vleeschouwer et al., 2007). Este grupo de descriptores muestran propiedades globales para el sistema como un todo. Sin embargo, para estudiar la selectividad y reactividad de las especies químicas se hace necesario usar descriptores locales reactividad. La función de Fukui (Fuentealba et al., 2010) corresponde a un descriptor local y se define como:

$$
f(r)=\left(\frac{\partial \rho(r)}{\partial N}\right)_{v(r)}=\left(\frac{\delta \mu}{\delta v(r)}\right)_{N}
$$

Esta función puede ser interpretada como la variación de la función de densidad electrónica ( $\rho)$, cuando el número de electrones cambia; es decir, indica la tendencia de la densidad electrónica a deformarse en una posición dada, bien sea aceptando o donando electrones (Parr y Weitao, 1994). De la ecuación 4, también se puede afirmar que el ataque de un reactivo sobre sitios específicos en una molécula, corresponderá a aquellos donde $f(r)$ es alta. En este trabajo, solamente se utilizó la función de Fukui para el ataque nucleofílico debido a la interacción de los colorantes con especies nucleofílicas. Por lo tanto, la función de Fukui de interés viene dada por:

$$
f^{+}(r)=\left(\frac{\partial \rho(r)}{\partial N}\right)_{v(r)}
$$

Que cuando se condensa a átomos, para un átomo j, viene dada por:

$$
f^{+}(r)=q_{j}(N+1)-q_{j}(N)
$$

En la ecuación 6, el término $q_{j}$ es la población electrónica del átomo en la molécula conteniendo el número de electrones mencionados entre paréntesis, donde $q_{j}(N+1)$ representa la especie aniónica y $\mathrm{q}_{\mathrm{j}}(\mathrm{N})$, la especie neutra (Mendoza y Ríos, 2011). Se ha reportado que, para la función de Fukui, su valor es completamente dependiente del tipo de carga usada y la mayoría de los métodos empleados para el cálculo de las cargas, están basados en algún tipo de análisis de población (Saha et al., 2009). El análisis de población natural (NBO, por sus siglas en inglés), permite calcular la función de Fukui con buena aceptación (Nazari y Zali, 2007). Con respecto al índice de electrofilicidad local, se puede calcular a partir de una expresión recientemente definida para un sitio j en una molécula (Chattaraj et al., 2003; Sharma, 2009), a través de la ecuación (7). La introducción de este concepto, permite analizar las reacciones donde se dan ataques nucleofílicos como es el caso de los sistemas estudiados aquí.

$$
\omega_{j}^{+}=\omega f_{j}^{+}
$$

Donde, $\mathrm{f}_{\mathrm{j}}^{+}$es la función de Fukui para el ataque nucleofílico y $\omega$ es el índice de electrofilicidad global.

\section{Detalles Computacionales}

Las geometrías de los colorantes rojo allura y amarillo anaranjado fueron completamente optimizadas en fase gas sin restricciones a la geometría empleando el funcional híbrido B3LYP y el conjunto de funciones base $6-31 \mathrm{G}(\mathrm{d})$, implementados en el paquete de programas Gaussian-03 (Frisch, 2004) . A partir de las geometrías optimizadas en fase gas, se corrieron cálculos usando el modelo de solvente del continuo polarizado (Mennucci y Cammi, 2007) al mismo nivel de teoría, con el fin de simular la fase acuosa. Fue usada una constante dieléctrica de 78.5 a una 
temperatura de $25^{\circ} \mathrm{C}$. También, se realizaron cálculos de frecuencias vibracionales para corroborar que los puntos estacionarios localizados sobre la superficie de energía potencial B3LYP correspondían a mínimos (valores positivos de la matriz hessiana). Los descriptores globales de la reactividad fueron calculados usando los orbitales frontera HOMO (orbital molecular ocupado de más alta energía) y LUMO (orbital molecular desocupado de más baja energía) respectivamente. La función condensada de Fukui, para el ataque nucleofílico se calculó a través de la ecuación (6). El índice de filicidad local, fue calculado por medio de la ecuación (7) relacionando las cantidades globales $\omega$ con la función de Fukui para el ataque nucleofílico $\left(f^{+}{ }_{j}\right)$ sobre cada sitio j de la molécula.

\section{RESULTADOS Y DISCUSIÓN}

En la figura 1, se presentan las geometrías optimizadas en fase gas al nivel B3LYP/6-31G(d) para los colorantes amarillo anaranjado y rojo allura respectivamente.

A

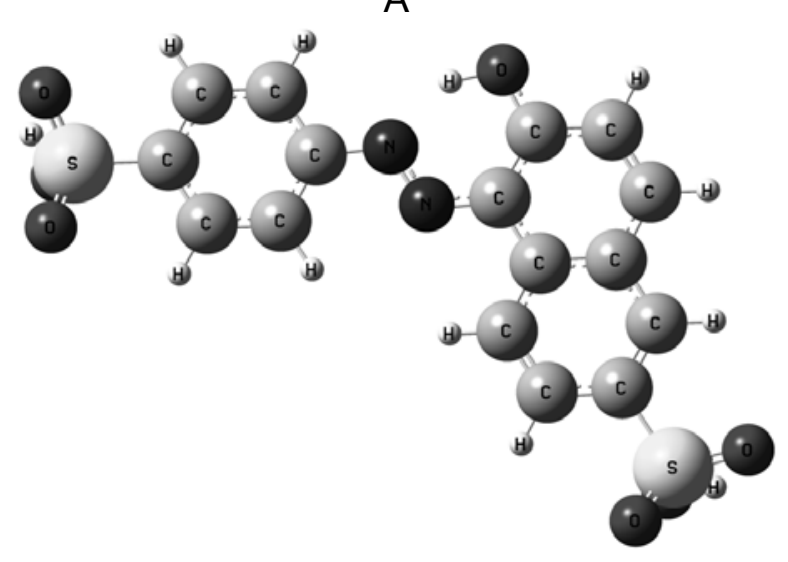

B

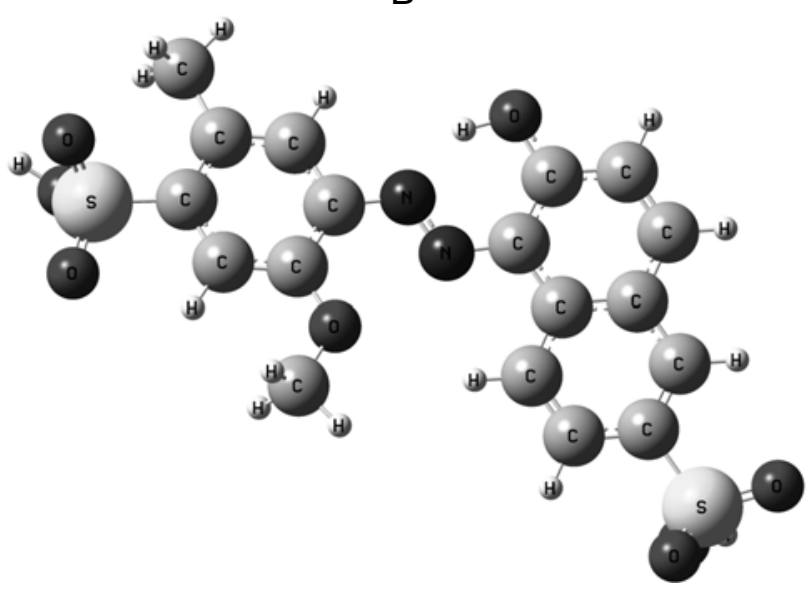

Fig.1: Estructuras optimizadas en fase gas para los colorantes amarillo anaranjado $(A)$ y rojo allura (B) al nivel B3LYP/6-31G(d)

En la tabla 1, se presentan los valores calculados para los descriptores globales de la reactividad en vacio y en fase acuosa al mismo nivel de teoría. De acuerdo con los datos de la tabla 1, se puede notar que, los descriptores globales para ambos colorantes, tienen valores muy cercanos en las dos fases lo cual se debe en parte a su similitud estructural, siendo claro que la naturaleza reactiva de estas especies resulta parecida. No obstante, si se tiene en cuenta el principio de máxima dureza (Pearson, 2005; Parr y Chattaraj, 1991), se observa que en la fase acuosa amarillo anaranjado es levemente más estable que rojo allura y por ende se esperaría que sea menos reactivo. Sin embargo, el índice de electrofilicidad para amarillo anaranjado es más alto que para rojo allura y por lo tanto tiene mayor capacidad para recibir los electrones provenientes del agente reductor bisulfito de sodio. Así, es evidente que, el azo colorante amarillo anaranjado es más susceptible a la adición nucleofílica con respecto a rojo allura. Ahora bien, la disminución del valor en el índice de electrofilicidad para rojo allura, se debe a la presencia de los sustituyentes metilo $\left(-\mathrm{CH}_{3}\right)$ y metoxilo $\left(-\mathrm{OCH}_{3}\right)$ sobre el grupo funcional sulfofenil, ya que debido a su carácter electro donador, ceden densidad electrónica a la molécula, implicando con ello una disminución en la capacidad del grupo azo para aceptar electrones provenientes del agente reductor, de modo que, la especie es menos susceptible a la adición nucleofílica del ión bisulfito en relación con amarillo anaranjado. El leve incremento de la dureza en la fase acuosa, indica que este medio estabiliza los colorantes. Además cabe notar que, el potencial químico $(\mu)$ y el índice de electrofilicidad $(\omega)$, para ambos colorantes disminuyen en presencia de agua y como es lógico, si la estabilidad aumenta la reactividad de las moléculas disminuye. Por otro lado, en la literatura científica se ha reportado con suficiencia que la estabilidad de los azo colorantes frente al ión bisulfito es variable y los procesos de decoloración pueden ocurrir a través de dos vías (Adams y Langley, 1995). Es así como se ha observado que, para los colorantes amarillo anaranjado, amaranto $(E-123, C . I .16185)$ y punzó $4 R(E-124, C . I .16255)$ la reacción con bisulfito genera un color secundario debido a la aparición de un producto que absorbe a diferente longitud de onda. 
Así por ejemplo, el colorante amarillo anaranjado se degrada a un compuesto de color amarillo limón a una longitud de onda máxima de $418 \mathrm{~nm}$ (Gameay, 2002). Con respecto a la segunda vía, ocurre la decoloración pero sin cambios en la longitud de onda de absorción máxima y sin la aparición de colores secundarios como sucede para tartrazina (E-102, C.I.19140) y azorrubina (E122, C.I.14720) (Adams y Langley,1995).

Tabla 1: Descriptores globales de la reactividad para amarillo anaranjado y rojo allura calculados al nivel B3LYP/6-31G(d) en vacio y fase acuosa

\begin{tabular}{cccccc}
\hline \multicolumn{7}{c}{ FASE GAS } \\
\hline COLORANTE & $\varepsilon_{\mathrm{H}}(\mathrm{eV})$ & $\varepsilon_{\mathrm{L}}(\mathrm{eV})$ & $\mu(\mathrm{eV})$ & $\eta(\mathrm{eV})$ & $\omega(\mathrm{eV})$ \\
\hline Amarillo anaranjado & -6.45 & -3.27 & -9.71 & 1.59 & 29.64 \\
Rojo allura & -6.12 & -2.94 & -9.06 & 1.59 & 25.79 \\
\hline \multicolumn{5}{c}{ FASE ACUOSA } \\
\hline Amarillo anaranjado & -6.23 & -3.02 & -9.25 & 1.61 & 26.66 \\
Rojo allura & -6.04 & -2.86 & -8.90 & 1.59 & 24.87 \\
\hline
\end{tabular}

Además, para la primera vía por estudios cinéticos empleando técnicas espectrofotométricas convencionales se encontró que para los colorantes amarillo anaranjado y punzó 4R las reacciones son reversibles debido a la aparición de puntos isosbésticos en el espectro de absorción obtenido (Gameay, 2002). Por lo tanto, se asume que existe un equilibrio tautomérico entre las especies azo e hidrazona de estos colorantes. La presencia de la especie hidrazona en solución acuosa facilita la adición nucleofílica del ión bisulfito sobre el azo colorante en la posición 4 (figura 2), ya que se ha reportado que esta posición es más accesible debido a su menor impedimento estérico con respecto a la posición 2 (C2) (Damant et al.,1989; Berke et al.,1998). Por esta razón, también se realizaron cálculos computacionales sobre las especies hidrazona de amarillo anaranjado $\left(\mathrm{AN}_{2}\right)$ y rojo allura $\left(\mathrm{RA}_{2}\right)$ con el fin de evaluar su reactividad. Así, en la tabla 2, se presentan los descriptores globales de la reactividad obtenidos para las especies hidrazona en vacio y fase acuosa. Como se esperaba, efectivamente las formas hidrazona presentan un mayor $\omega$ y menor $\eta$ que las especies tautoméricas azo (figura 1), sugiriendo que su comportamiento electrofílico se incrementa, al mismo tiempo que su reactividad frente al ión bisulfito también lo hace. En resumen, las especies tautoméricas hidrazona mostraron un incremento en su comportamiento electrofílico con respecto a las especies azo, que es un resultado en buen acuerdo con la evidencia experimental (Damant et al., 1989; Gemeay, 2002). Por otro lado, aunque se mostró por vía experimental que la adición nucleofílica del ión bisulfito ocurre sobre C4 (figura 2), no hay estudios computacionales al respecto y en este sentido, un análisis de la selectividad de sitio en el contexto de los descriptores locales de la reactividad resultaría interesante. Por ello, se llevaron a cabo cálculos computacionales de la función nucleofílica de Fukui e índice de electrofilicidad local y los principales sitios reactivos de las moléculas investigadas son mostrados en la figura 3. Para mayor claridad el núcleo naftalénico ha sido numerado. Además en la tabla 3 , se muestran los valores calculados para los descriptores locales de la reactividad.

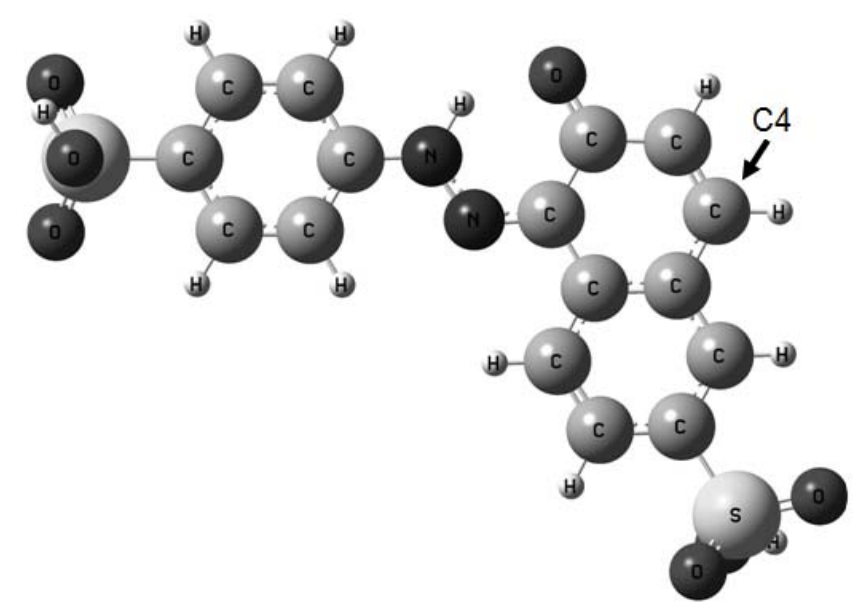

Fig.2: Geometría optimizada para la especie hidrazona de amarillo anaranjado en fase gas 
Tabla 2: Descriptores globales de la reactividad para las especies hidrazona de amarillo anaranjado y rojo allura calculados al nivel B3LYP/6-31G(d) en vacio y fase acuosa

\begin{tabular}{cccccc}
\hline \multicolumn{7}{c}{ FASE GAS } \\
\hline COLORANTE & $\varepsilon_{\mathrm{H}}(\mathrm{eV})$ & $\varepsilon_{\mathrm{L}}(\mathrm{eV})$ & $\mu(\mathrm{eV})$ & $\eta(\mathrm{eV})$ & $\omega(\mathrm{eV})$ \\
\hline $\mathrm{AN}_{2}$ & -6.42 & -3.29 & -9.71 & 1.56 & 30.16 \\
$\mathrm{RA}_{2}$ & -6.04 & -3.02 & -9.06 & 1.51 & 27.18 \\
\hline \multicolumn{5}{c}{ FASE ACUOSA } \\
\hline $\mathrm{AN}_{2}$ & -6.20 & -3.07 & -9.28 & 1.56 & 27.51 \\
$\mathrm{RA}_{2}$ & -5.96 & -2.94 & -8.90 & 1.51 & 26.21 \\
\hline
\end{tabular}

Tabla 3: Función nucleofílica de Fukui $\left(\mathrm{f}^{+}(\mathrm{r})\right.$, en u.a), e índice de electrofilicidad local ( $\omega_{\mathrm{j}}^{+}$en eV) para las especies tautoméricas $\mathrm{AN}_{2}$ y $R A_{2}$ al nivel $B 3 L Y P / 6-31 G(d)$

\begin{tabular}{cccc|cc}
\hline & & \multicolumn{2}{c|}{$\mathrm{f}^{+}(\mathrm{r})(\mathrm{u} . \mathrm{a})$} & \multicolumn{2}{c}{$\mathrm{\omega}_{\mathrm{j}}^{+}(\mathrm{eV})$} \\
\hline Especie & Átomo & Vacio & Fase acuosa & Vacio & Fase acuosa \\
\hline & C1 & -0.011 & -0.022 & -0.33 & -0.61 \\
$\mathrm{C} 2$ & -0.063 & -0.079 & -1.90 & -2.17 \\
$\mathrm{AN}_{2}$ & $\mathrm{C} 3$ & -0.009 & -0.015 & -0.27 & -0.41 \\
& $\mathrm{C} 4$ & -0.078 & -0.079 & -2.35 & -2.17 \\
& $\mathrm{C} 5$ & -0.012 & -0.012 & -0.36 & -0.33 \\
& C6 & -0.038 & -0.031 & -1.15 & -0.85 \\
& C7 & -0.047 & -0.042 & -1.42 & -1.16 \\
& C8 & -0.006 & -0.012 & -0.18 & -0.33 \\
\hline & C1 & -0.006 & -0.016 & -0.16 & -0.42 \\
& C2 & -0.063 & -0.076 & -1.71 & -1.99 \\
& C3 & -0.012 & -0.016 & -0.33 & -0.42 \\
$\mathrm{RA}_{2}$ & C4 & -0.075 & -0.078 & -2.04 & -2.04 \\
& C5 & -0.012 & -0.013 & -0.33 & -0.34 \\
& C6 & -0.034 & -0.029 & -0.92 & -0.76 \\
& C7 & -0.046 & -0.043 & -1.25 & -1.13 \\
& C8 & -0.003 & -0.009 & -0.08 & -0.24 \\
\hline
\end{tabular}

Como se indicó previamente, la dirección preferida de ataque del ión bisulfito sobre los sitios indicados en la figura 3, corresponden a aquellos donde la variación del potencial químico electrónico es máxima, es decir, aquellos sitios para los cuales la función condensada de Fukui $\left(\mathrm{f}_{\mathrm{j}}^{+}\right)$es alta. De este modo, se observa que para los tautómeros hidrazona de ambos colorantes, el átomo C4 presentó el mayor valor de la función Fukui tanto en fase gas como en la fase acuosa (tabla 3), seguido del átomo C2. Estos resultados mostraron buena concordancia con la evidencia experimental, pues la selectividad de sitio para el ión bisulfito, efectivamente se dió sobre C4. Por otro lado la posición C2, de acuerdo con lo reportado en la literatura se encuentra impedida en relación con la posición $\mathrm{C} 4$, debido a la electrostática y repulsión estérica del átomo de oxígeno, de ahí que, el valor obtenido para $\mathrm{f}_{j}^{+}$fuera sutilmente menor. También, se puede notar que, $\mathrm{f}^{+}{ }_{j}$ del átomo $\mathrm{C} 4$ en $\mathrm{AN}_{2}$ es levemente mayor que en $\mathrm{RA}_{2}$, confirmando que la especie $\mathrm{AN}_{2}$ es más susceptible a la adición del ión bisulfito, lo cual es una consecuencia directa de la presencia de los grupos electrodonadores metilo y metoxilo en rojo allura. Otro aspecto a considerar mostrado en la tabla 3, son los valores negativos para la función de Fukui (Melin, 2007) y que de acuerdo a lo planteado por Ayres (Ayers et al., 2002), se pueden relacionar con la reducción u oxidación de los centros atómicos en la molécula. Además, recientemente se reportó que cuando se adiciona un electrón a una molécula en algunos sitios de ésta, la densidad electrónica se reduce (Moncada y Salgado, 2007), como es el caso de las moléculas rojo allura y amarillo anaranjado. Ahora bien, de estos resultados también se hace evidente que en un sentido global, los sitios más reactivos de $\mathrm{AN}_{2}$ y $R A_{2}$, están directamente relacionados con aquellos átomos que contribuyen con el orbital 
LUMO, ya que, se esperaría que el exceso de electrones provenientes del agente reductor (ión bisulfito), se localice sobre los átomos con mayores coeficientes LUMO.

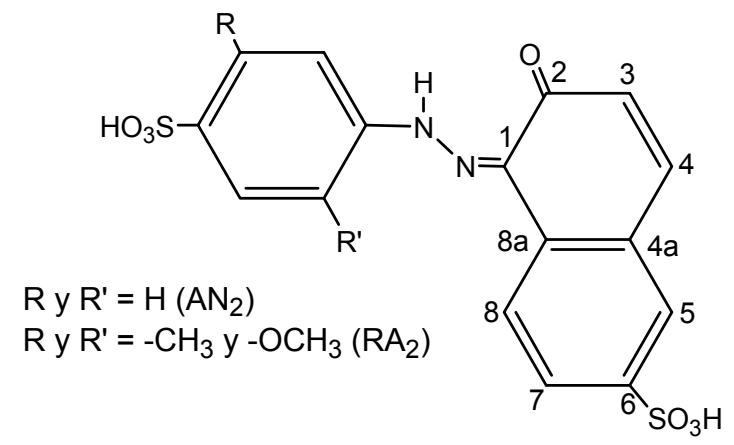

Fig. 3: Átomos numerados sobre el núcleo naftalénico en los colorantes estudiados

Así por ejemplo, en la figura 4, se presenta el gráfico de contorno para el orbital frontera LUMO, de la especie $\mathrm{AN}_{2}$, donde se observa que el LUMO está localizado sobre el núcleo naftalénico. La contribución del LUMO en C4, es significativa y por lo tanto se confirma su tendencia de llevar a cabo la adición nucleofílica.

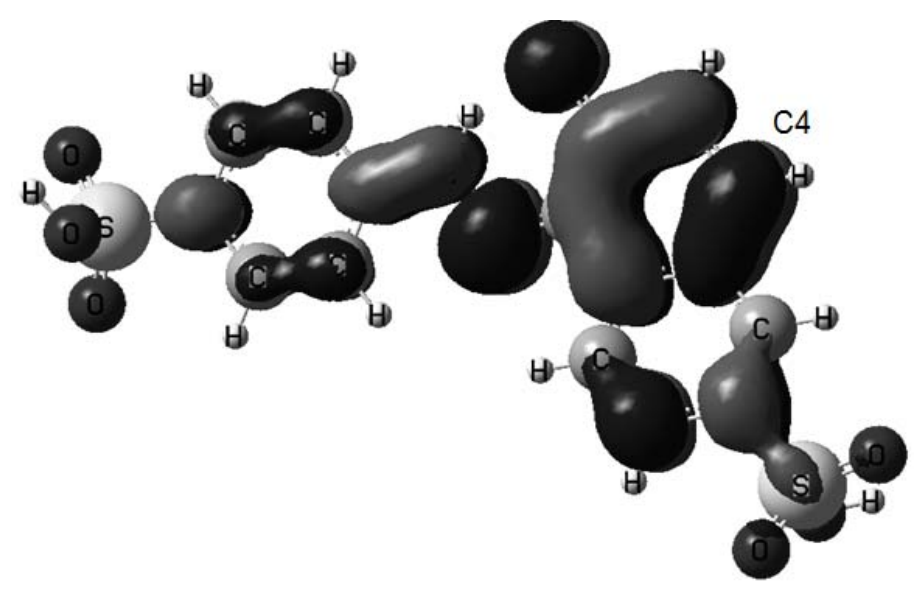

Fig.4: Superficie del orbital molecular LUMO calculada al nivel B3LYP/6-31G(d) con un valor de isosuperficie de $0.03 \mathrm{e}^{-u^{3}}$

Por otro lado, el índice de electrofilicidad local, presentó información similar a la función nucleofílica de Fukui, confirmando los resultados.

\section{CONCLUSIONES}

Con base en los resultados obtenidos del estudio se puede decir que los descriptores de la reactividad globales y locales derivados de la teoría del funcional de la densidad, permitieron hacer una descripción cualitativa de la reactividad química y estabilidad relativa de los colorantes amarillo anaranjado y rojo allura. El análisis de la función condensada de Fukui para el ataque nucleofílico e índice de electrofilicidad local, mostraron que el sitio preferido de ataque del agente reductor (ión bisulfito) se da sobre $\mathrm{C} 4$, lo cual estuvo en concordancia con la literatura. Además, los sitios más reactivos sobre el núcleo naftalénico están relacionados con aquellos átomos que contribuyen con el orbital LUMO ya que se esperaría que el exceso de electrones provenientes del agente reductor (ión bisulfito) se localice sobre los átomos con mayores coeficientes LUMO.

\section{AGRADECIMIENTOS}

Al Centro de Investigaciones de la Universidad de Córdoba (CIUC) por financiar el proyecto FCB07-08 numeral 1120272. Al estudiante de Doctorado en The Beacon Partnership, Julio Román Maza Villegas por sus valiosos aportes en el desarrollo de este manuscrito. 


\section{REFERENCIAS}

Adamson, P.E., X.F., Duan y L.W. Burgraff, Modeling Positrons in Molecular Electronic Structure Calculations with the Nuclear-Electronic Orbital Method, J. Phys. Chem. A: 112(6), 1346-1351 (2008).

Adams, J.B. y F.M. Langley, Interaction between additives in food systems, Campden \& Chorleywood Food Research Association, (1995).

Ayers, P.W., R.C. Morrison y R.K. Roy, Variational principles for describing chemical reactions: Condensed reactivity indices, J. Chem. Phys: 116(20), 8731-8744 (2002).

Berke B., C. Cheze, J. Vercauteren y G. Deffieux, Bisulfite addition to anthocyanins: revisited structures of colourless adducts, Tetrahedron Lett: 39(32), 5771-5774 (1998).

Chattaraj, P.K. y S. Giri, Electrophilicity index within a conceptual DFT framework, Annu. Rep. Prog. Chem. Sect. C: Phys. Chem: 105(0), 13-39 (2009).

Chattaraj, P.K., B. Maiti, y U. Sarkar., Philicity, A Unified Treatment of Chemical Reactivity and Selectivity. J. Phys. Chem. A: 107(25), 4973-4975 (2003).

Damant, A., S. Reynolds y R. Macrae, The structural identification of a secondary dye produced from the reaction between sunset yellow and sodium metabisulphite, Food. Addit. Contam: 6(3), 273-282 (1989).

De Vleeschouwer, F., y Otros, Electrophilicity and Nucleophilicity Index for Radicals, Org. Lett: 9(14), 2721-2724 (2007).

Esquivel, R. y Otros, Phenomenological Description of a Three-Center Insertion Reaction: An Information-Theoretic Study, J. Phys. Chem. A: 114(4), 1906-1916 (2010).

Frisch, M. J., G. W. Trucks y H.B. Schlegel., Gaussian 03, Wallingford CT, Gaussian, Inc, (2003). Fuentealba, P., E. Florez y W. Tiznado, Topological Analysis of the Fukui Function, J. Chem. Theory Comput: 6(5), 1470-1478 (2010).

Gemeay, A.H., Kinetics and mechanism of the reduction of some azo-dyes by inorganic oxysulfur compounds, Dyes Pigments: 54(3), 201-212 (2002).

Mendoza, L. y C. Ríos, Chemical Reactivity of Atrazine Employing the Fukui Function, J. Mex. Chem. Soc: 55(3), 142-147 (2011).

Melin, J., P.W., Ayers y J.V. Ortiz, Removing Electrons Can Increase the Electron Density: A Computational Study of Negative Fukui Functions, J. Phys. Chem. A: 111(40),10017-10019 (2007).

Moncada, L y G. Salgado, Caracterización de la reactividad intrínseca de los halobencenos en el modelo conceptual de la teoría de funcionales de la densidad (TFD), An. RSEQ: (3), 23-27 (2007).

Marmion, D.M., Handbook of U.S. Colorants: Foods, Drugs, Cosmetics, and Medical Devices, $3^{\text {a }}$ edición, pp 350, Wiley-Interscience, One Wiley Drive, Somerset, NJ 08873, (1979).

Mennucci, B. y R. Cammi, Continuum solvation models in chemical physics: from theory to applications, John Wiley \& Sons, Ltd, Chichester, UK, (2007).

Nazari, F. y Z.F. Rasoulzadeh, Density functional study of the relative reactivity of the carbonyl group in substituted cyclohexanone, J Mol. Struc-Theochem: 817(1-3), 11-18 (2007). 
Nelsen, S.F. y Otros, Calculations of the Optical Spectra of Hydrocarbon Radical Cations Based on Koopmans' Theorem, J. Phys. Chem. A: 111(9), 1667-1676 (2007).

Özen, A. S., V. Aviyente y R.A. Klein, Modeling the Oxidative Degradation of Azo Dyes: A Density Functional Theory Study, J. Phys. Chem. A: 107(24), 4898-4907 (2003).

Özen, A.S, y V. Aviyente, Modeling the Substituent Effect on the Oxidative Degradation of Azo Dyes, J. Phys. Chem. A: 108(28), 5990-6000 (2004).

Parr, R.G. y P.K. Chattaraj, Principle of maximum hardness. J. Am. Chem. Soc: 113(5), 18541855 (1991).

Parr, R.G. y Y.Weitao, Density-Functional Theory of Atoms and Molecules, Oxford University Press, USA, (1994).

Pearson, R.G., The Principle of Maximum Hardness in Chemical Hardnes, Wiley-VCH Verlag GmbH \& Co, KGaA, Weinheim FRG, 99-124 (2005).

Plakhutin, B.N. y E.R. Davidson, Koopmans' Theorem in the Restricted Open-Shell Hartree-Fock Method. 1. A Variational Approach, J. Phys. Chem. A: 113(45), 12386-12395 (2009).

Saha, S., R. Roy y P. Ayers, Are the Hirshfeld and Mulliken population analysis schemes consistent with chemical intuition?, Int. J. Quantum. Chem: 109(9), 1790-1806 (2009).

Sharma, P., A. Kumar y V. Sahu, Theoretical Evaluation of Global and Local Electrophilicity Patterns to Characterize Hetero-Diels-Alder Cycloaddition of Three Membered 2H-Azirine Ring System, J. Phys. Chem. A: 114(2), 1032-1038 (2010).

Scotter, M.J. y L. Castle, Chemical interactions between additives in foodstuffs: a review, Food Add. Contam: 21(2), 93-124 (2004).

Umer Abdullah, S. M. Badaruddin, S.A. Sayed, R. Ali y M.N. Rian, Binding ability of Allura Red with food proteins and its impact on protein digestibility, Food Chem: 110(3), 605-610 (2008).

Ustun, M., A Rapid Spectrophotometric Method to Resolve a Binary Mixture of Food Colorants (Riboflavine and Sunset Yellow), Turk. J Chem: 28(3), 325-333 (2004).

Vivas-Reyes, R., F. Núñez-Zarur y E. Martínez, Electronic structure and reactivity analysis for a set of Zn-chelates with substituted 8-hydroxyquinoline ligands and their application in OLED, Org. Electron: 9(5), 625-634 (2008).

Wedzicha, B.L. y S.J. Rumbelow, The reaction of an azo food dye with hydrogen sulphite ions, J Sci Food Agric: 32(7), 699-704 (1981).

Winkler, C., B. Frick, K. Schroecksnadel, H. Schennach y D. Fuchs, Food preservatives sodium sulfite and sorbic acid suppress mitogen-stimulated peripheral blood mononuclear cells, Food Chem. Toxicol: 44(12), 2003-2007 (2006). 
